${ }^{3}$ Diabetic medicine

${ }^{4}$ Cleveland Clinic Advanced Certificate Courses in Diabetes, New Delhi India

\title{
A review on generation of real-world evidence
}

\section{ABSTRACT}

Real-world evidence can generate credible evidence to inform treatment decisions. Real-world evidence is in developmental stage and is fast evolving yet there are many unexplained attributes of real-world evidence. Real-world evidence informs benefit-risk decisions and is increasingly being used to support regulatory decision making. Potential benefits of real-world data include determination of extended outcomes including long-term outcomes, opportunities to partner with patients in innovative ways, and reduction in time and cost to generate dependable evidence. Limitations of real-world evidence include uncertainty in the quality of datasets and lack methodologic rigor in real-world studies. Use of real-world evidence for healthcare practices and policies is limited. Ensuring completeness, homogeneity, and linkage of datasets can enhance utility for epidemiological investigations and improvement in health outcomes. Research should be strengthened for real-world studies and technical standards should be reinforced. Collaborations of stakeholders is key to formulation and adoption of guidance for real-world evidence. Real-world data cannot be a substitute to randomized clinical studies but can possibly augment the generated evidence. (Clin Diabetol 2021; 10; 5: 412-419)

Key words: real-world evidence, healthcare policies, real-world data, pragmatic trials, policy making

Address for correspondence:

M.S. Shunmugavelu

Trichy Diabetes Specialty Centre

Trichy, Tamilnadu, 620018

e-mail: info@mediception.com

Clinical Diabetology 2021, 10; 5: 412-419

DOI: $10.5603 /$ DK.2021.0049

Received: 7.01.2020

Accepted: 8.03.2021

\section{Introduction}

Medical practices are adopting evidence-based trends and real-world evidence (RWE) is gaining attention and importance. Real-world data (RWD) is increasingly becoming the intellectual capital of healthcare. RWD are patient health related data and/or health care delivery, routinely collected from varied sources. RWE is the evidence for usage, that informs about disease epidemiology, treatment patterns, safety and efficacy of therapies, and approval and reimbursement of drugs. Trends in RWE have gained momentum since 2016 when the United States Food and Drug Administration (USFDA) notified the consideration of RWD in regulatory decision-making [1, 2]. In this review, we discuss the types and sources for RWD, differentiation of real-world studies from randomized controlled trials (RCTs), advantages and limitations of RWE, perceptions and regulatory perspectives for RWE, and the challenges of adoption of RWE in India.

\section{Definition of real-world evidence}

A standard definition of RWE is lacking though there is evident zeal to adopt RWE for advancement in healthcare [3]. The USFDA has defined RWD as "data regarding the usage, or the potential benefits or risks, of a drug derived from sources other than traditional clinical trials" and RWE as "clinical evidence about the usage and potential benefits or risks of a medical product derived from analysis of RWD" [4].

RWE is generated by disparate study designs or analyses, that among others includes, randomized trials, large simple trials, pragmatic trials, and observational studies. RWD and RWE are increasingly influencing health care decisions [5].

\section{Randomized controlled and real-world studies}

RCTs have been regarded as a gold standard of evidence. However, RCTs have inherent limitations 
Table 1. Comparison of randomized controlled and real-world clinical studies

\begin{tabular}{|c|c|c|}
\hline Parameters & Clinical trial & Real-world studies \\
\hline Patient population & Homogenous & Heterogenous \\
\hline Study design & $\begin{array}{c}\text { Controlled and defined clinical settings } \\
\text { driven by protocol }\end{array}$ & $\begin{array}{c}\text { Driven by routine clinical practices, } \\
\text { no comparator or control }\end{array}$ \\
\hline Therapeutic utilization & Protocol driven dosing and monitoring & Real-world dosing patterns and challenges \\
\hline Key regulatory purpose & $\begin{array}{l}\text { Requirement for efficacy and safety } \\
\text { for drug approval }\end{array}$ & $\begin{array}{c}\text { Can confirm the efficacy and safety in wider } \\
\text { patient populations after approval }\end{array}$ \\
\hline Value for health economy & Very limited use & Widely used \\
\hline Acceptance by payers & $\begin{array}{l}\text { Traditional requirement for health technology } \\
\text { assessment and pricing }\end{array}$ & $\begin{array}{l}\text { Increasing acceptance by payers and health } \\
\text { technology assessment bodies }\end{array}$ \\
\hline Component in drug development & Phases of clinical trials are well defined & $\begin{array}{l}\text { Span the entire spectrum of product life cycle } \\
\text { from development to post authorization safety }\end{array}$ \\
\hline
\end{tabular}

including controlled settings in a predefined sample that may not be representative of the entire universe. Evidence of safety and efficacy in RCTs cannot always be generalized which is explained by the focus of internal validity in RCTs. Evidence from RCTs may not adequately inform about clinical practice guidelines. Clinical practice challenges may require decisions for dosing and assessment of effectiveness in diverse patient populations $[2,6,7]$. There is now an evolving trend to generate and recognize evidence in real-world settings. RCTs and real-world studies have discrete differences. RCTs are interventional with targeted objectives for efficacy and safety whereas RWE studies are observational and focus on effectiveness and economic assessments [8]. Effectiveness is different from efficacy as the assessment is targeted to determine if the intervention does more good than harm when used in the circumstances of a routine healthcare practice. Efficacy, on the other hand, defines the extent of good under ideal and controlled circumstances [9]. FDA passed the $21^{\text {st }}$ Century Cures Act on December 8, 2016, and following that released eight publications. The articles asserted that the principal attribute distinguishing realworld evidence is the context of accumulated evidence. RWE employs clinical, home or community setting in contrast to the research-oriented background of RCTs [10]. The key differences of RCTs and RWE studies are shown in Table $1[3,8,11,12]$

\section{Need for real-world evidence}

RWE is increasingly being used for healthcare decisions. RWE provides an opportunity to build valuable evidence which cannot be generated using the traditional RCTs. RWEs provide evidence for characteristics that are outside the realm of those typically required for trial eligibility. RWE can inform benefits and risks of a particular treatment with more focus on patient characteristics. RWE spans to heterogenous populations with diverse demographic characteristics, those with comorbidities and receiving concomitant medications, and people with limited social resources. It is essentially about getting the right treatment to the right patient at the right time. RWE provides insights for the natural progression of the disease in realistic and uncontrolled settings. RWE is the basis for building a more patientcentric approach in healthcare.

\section{Types and sources of real-world evidence}

RWE is an integration of diverse and multiple data sources such as, epidemiological studies, surveillance studies, healthcare records, and data from administrative databases and personal devices [13]. Studies using one or many data sources for RWE studies should be well- designed and properly executed. It is prudent to develop a protocol with a clear and sound methodology that can clearly define the objectives and answer the research questions.

RWE studies are classified as observational studies, safety studies, pragmatic trials, and surveys. Observational studies include both prospective and retrospective studies. Registries, health surveys, and post authorization safety studies are prospective studies. Retrospective studies include reviews of hospital charts, electronic medical records, and claims databases. Retrospective real-world studies are low-cost studies and easier to conduct. However, there are limitations of incomplete and inaccurate data. On the other hand, prospective studies are valuable to gather long-term data for disease course or progression. There is a higher cost associated with these studies and the duration depends on the recruitment of adequate samples to help answer the research questions [8]. The basic requirements for planning and design of research studies include accessibility, affordability, accuracy, advantage 


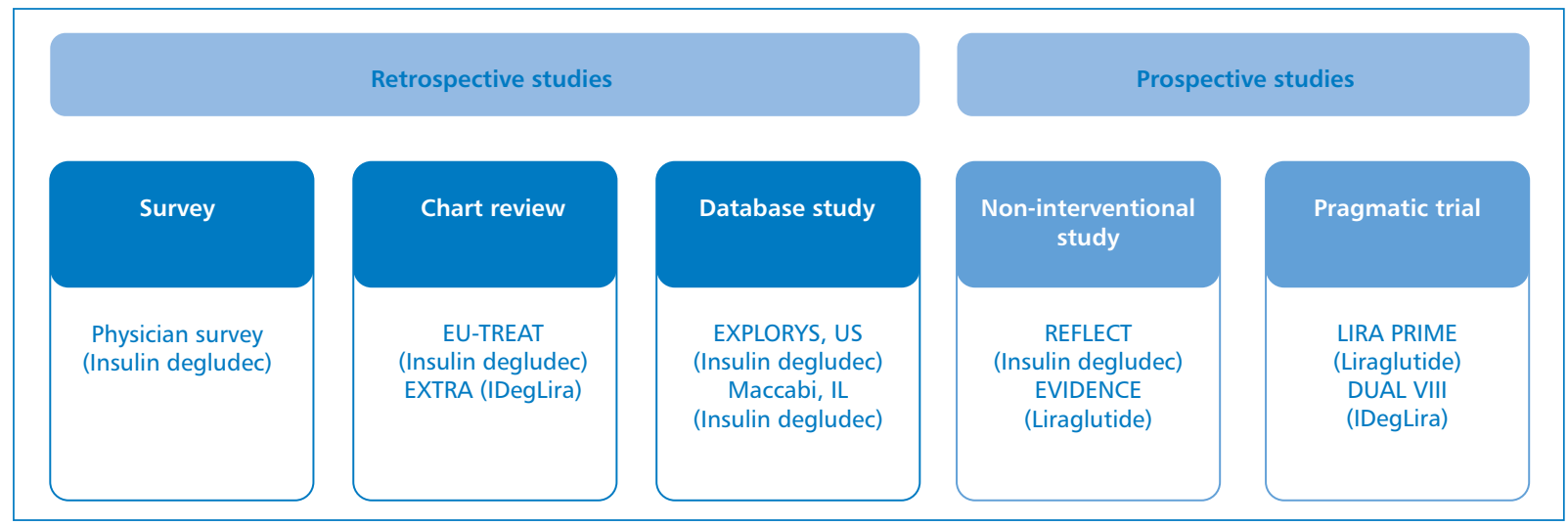

Figure 1. Types of real-world evidence

or appropriateness, attractiveness, appreciation or awareness, and acceptability [14]. Figure 1 shows the types of studies for RWE [12, 15-22].

Observational studies: Observational studies include both prospective and retrospective cohort studies. Electronic health records (EHRs) and medical claims and billing data are common datasets used for RWE [2]. Strong evidence can be generated from electronic healthcare databases (EHDs) which have data pools for huge number of patients over extensive periods and include a diverse patient population. In a recent study in 34 EHDs (Electronic medical records: $44.1 \%$, record linkage systems: $29.4 \%$, claims databases: $26.5 \%$ ), median number of registered patients was 5 million and the median time covered by a database was 18.5 years. A total of 32 databases (94\%) included pediatric patients [23]. The use of EHDs can provide valuable insights to encourage development of drugs in special populations like children, pregnant women, and elderly. This can help to mitigate the off-label use of medications in these populations.

Chart reviews: Retrospective chart reviews are based on pre-recorded patient-level data from more than one timepoint. Charts are cost-effective, easy to access and review. However, lack of external validity, diversity in patient populations and endpoints, and potential selection bias in comparison of two groups are some limitations of chart reviews. A retrospective, multicentre, chart review study (EU-TREAT) demonstrated the effectiveness of switching to insulin degludec (IDeg) in insulin-treated patients with type $1(n=1717)$ and type 2 ( $n=833$ ) diabetes [16].

Claims datasets: Retrospective studies from various datasets, e.g. claims data, are easy-to- conduct at low costs in large samples. Such studies, when conducted with a sound methodology, are credible. However, such datasets do not enable randomization and lend themselves to selection bias and potentially incompleteness of data. In a propensity-score- matched, retrospective, cohort study of the IMS PharMetrics claims data, health care resource

utilization and treatment patterns were compared in patients with actinic keratosis. Patients received treatment with Ingenol Mebutate gel (IngMeb) or other field directed therapies. In the first six months after initiation, treatment patterns were comparable for IngMeb and other therapies [24].

Other datasets: In another non-interventional, retrospective, pre-post cohort study, de-identified, EHR, medical billing, and payor claims data were retrieved from the IBM Explorys platform to enable real-world assessment of clinical effectiveness of switching to insulin degludec from another basal insulin in patients with type 2 diabetes [12]. A retrospective cohort study using data from the Clinical Practice Research Datalink (CPRD) database for 81573 patients with type 2 diabetes reported delays in treatment intensification despite suboptimal glycemic control [25]. Such studies provide insights for improvement of treatment practices.

Product and disease registries: Registries are nonintervention, prospective, observational studies with a predefined purpose of sustained and standardized data collection. They record and explore the treatment patterns in real-world and empower the patient and physician to take treatment and follow-up decisions. Registries find particular importance for assessment of natural history, real-world safety and effectives, prognosis and quality of life, and cost- effectiveness of treatment strategies. Inherent to design, registries lack the opportunity for randomization and have poor internal validity. Registries can be linked to other databases and enable the assessment of additional outcomes like healthcare utilization and mortality [26]. Patient registries are valuable tools in the conduct of research in 
several areas such as use of home oxygen therapy in patients with chronic obstructive pulmonary disease [27] and course of disease and treatment options in multiple sclerosis [28].

Safety studies: Safety assessments throughout the spectrum of drug development can be used to generate evidence that can help to safeguard the health and wellbeing of patients and provide objective and convincing considerations for payer and regulatory assessments [24]. A recent real-world study was done to establish the safety of direct oral anticoagulants and determine the risks of major bleeding, intra-cranial hemorrhage, gastrointestinal bleeding, and all-cause mortality. The RWE for safety was comparable to that reported in hallmark studies for the anticoagulants $[29,30]$.

Surveys: Surveys find utility for burden of disease studies. Surveys offer advantages of ease of conduct, possibility of multiple endpoints, administration in large samples, and low investments of time and money. However, surveys do not enable a follow-up and have a high risk of bias. An internet-based 20-minute survey was administered to 2108 patients with diabetes to determine the burden and impact of non-severe nocturnal hypoglycemic events on diabetes management, patient functioning and well-being [12]. This survey provided insights for patient and physician education for hypoglycemic events and corrective interventions for glycemic goals.

\section{Pragmatic clinical trials}

Pragmatic trials are conducted to demonstrate the real-world effectiveness, safety or health- economic benefits of a new intervention or a new indication for an established therapy. Pragmatic trials may include investigators who lack formal training in the conduct of medical research. By design, these trials have no defined frequency of follow-up visits, are influenced by the physician and patient attitude, acceptance of treatment, and inconsistencies in data collection. Pragmatic trials help to determine relative effectiveness and safety of a treatment option before or after market authorization [31]. The PRECIS-2 (PragmaticExplanatory Continuum Indicator Summary-2) tool is used to determine the level of pragmatism in a clinical study based upon parameters of recruitment of both, investigators and participants, study intervention, its delivery and follow-up, and the determination and analysis of outcomes [32]

The PRIDE study was a real-world study for superiority of paliperidone to oral antipsychotics in schizophrenia that led to label expansion by FDA in 2018 [33]. A metanalysis of retrospective and pragmatic real-world studies reported a discernible benefit of long-acting injectable (LAI) antipsychotics over oral antipsychotics in
Schizophrenia in terms of cost- effective and sustained patient outcomes [34].

\section{Real-world evidence and regulatory framework}

Regulators are beginning to recognize the value and usefulness of RWD to support applications for new indications. Efforts are being made to define the standards for RWE research and data generation [1]. A simple weighted RWD analysis supported the fasttrack approval of blinatumomab (Blincyto), a secondline treatment for Philadelphia chromosome- negative relapsed or refractory acute lymphoblastic leukemia, by the USFDA in 2018. Data supporting complete remission were gathered in follow up of adults enrolled in a single-arm phase III clinical trial of blinatumomab [35].

The US FDA has issued guidance for the use of RWE for drugs, biologics, and medical devices. The focus is on quality (relevance and reliability) of RWD and not study methodologies. (Available as: https://www.fda. gov/science-research/science-and-research- specialtopics/real-world-evidence) [36]. Regulatory uses of RWE include new indications for approved devices, shifts to pre- and post-market balance, use as control arm for pivotal clinical study, adding improvements in design of devices, replacing post-approval studies, and adverse event reporting. Pragmatic studies are being embraced by the European Medicines Agency. RCTs are moving closer to RWE with inclusion of estimands in regulatory guidelines. Estimands are used to measure treatment effects and possibility of effect under different treatment conditions [37]. By December 2021, the USFDA will publish draft guidance on how RWE can contribute to the assessment of safety and effectiveness in regulatory submissions [38].

\section{Conduct of real-world studies}

Real-world studies are conducted with diverse sources. A protocol should be prepared for any planned real-world study. The protocol has descriptions for the context and background, objectives, methodology, conduct, and ethical considerations of the study. The hypothesis to be tested, if any, should be clearly stated and a detailed statistical analysis plan should be developed. The research questions should be clearly defined, and the objectives should be specific, measurable, achievable, relevant, and time-based. The ethical considerations, including the details of benefits and risks, informed consent, and conduct of the study, should be elaborated. Finally, data may be analysed and reported in compliance with the protocol. Study results should be published for the dissemination of the findings from the study (Fig. 2). 


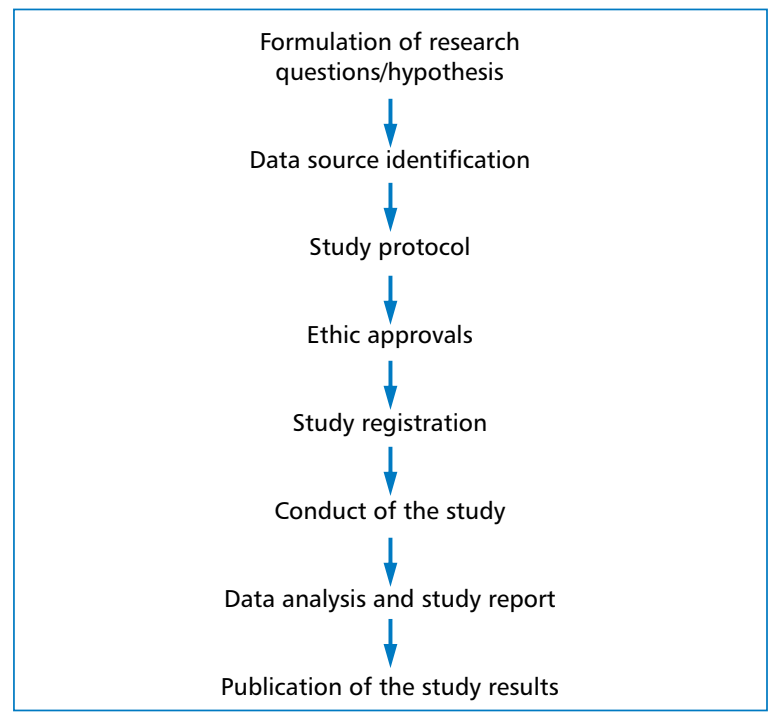

Figure 2. Steps for the conduct of real-world studies

\section{Applications and benefits of real-world evidence}

RWE can be used across the lifecycle of a product. The key utilities of RWE include the following:

1. Describe disease burden: RWD can provide insights for clinical, humanistic, and economic burden of diseases. RWD can be used to understand the natural history and course of disease, patient reported outcomes, healthcare utilization, and effectiveness of therapy [8].

2. Develop medical products: RWD can stimulate the generation of hypothesis and research questions to inform clinical development of medical products [15]. RCTs and RWE studies share a mutually supplementary relationship and when used together can foster evidence-based research [39].

3. Inform policy making in healthcare: The $21^{\text {st }}$ Century Cures Act (2016) has guidance for the US FDA to consider the use of RWE for approval of new indications for FDA- approved drugs [40].

4. Improve healthcare practices: Observational studies are used to monitor the safety of several products and devices. Patient registries can help in better diagnosis and treatment practices by providing insights from gathered patient data.

The ambit of RWD applications encompasses not only common diseases but also rare pathologies. It is unlikely for a primary care physician to witness a plethora of maladies in routine practice. Nevertheless, it is imperative to have sufficient and sound evidence to help make clinical decisions when encountering rare conditions. Real world data collated from the global health systems is an exciting prospect to strengthen clinical decision making [41]. RWE benefits a large segment of healthcare domain and society including the patients, physicians, payers, regulators, and drug developers (Fig. 3) [4, 40, 42, 43].

\section{The global and Indian perspective}

RWE has been utilized for healthcare decisions and advancements in the more developed world. Claims data have been used to build patient journeys by generating a longitudinal history through patient record linkage. Biomarker linkages have helped to gain precision in diagnosis, follow-up, and treatment in more targeted patient populations. Patient registries have

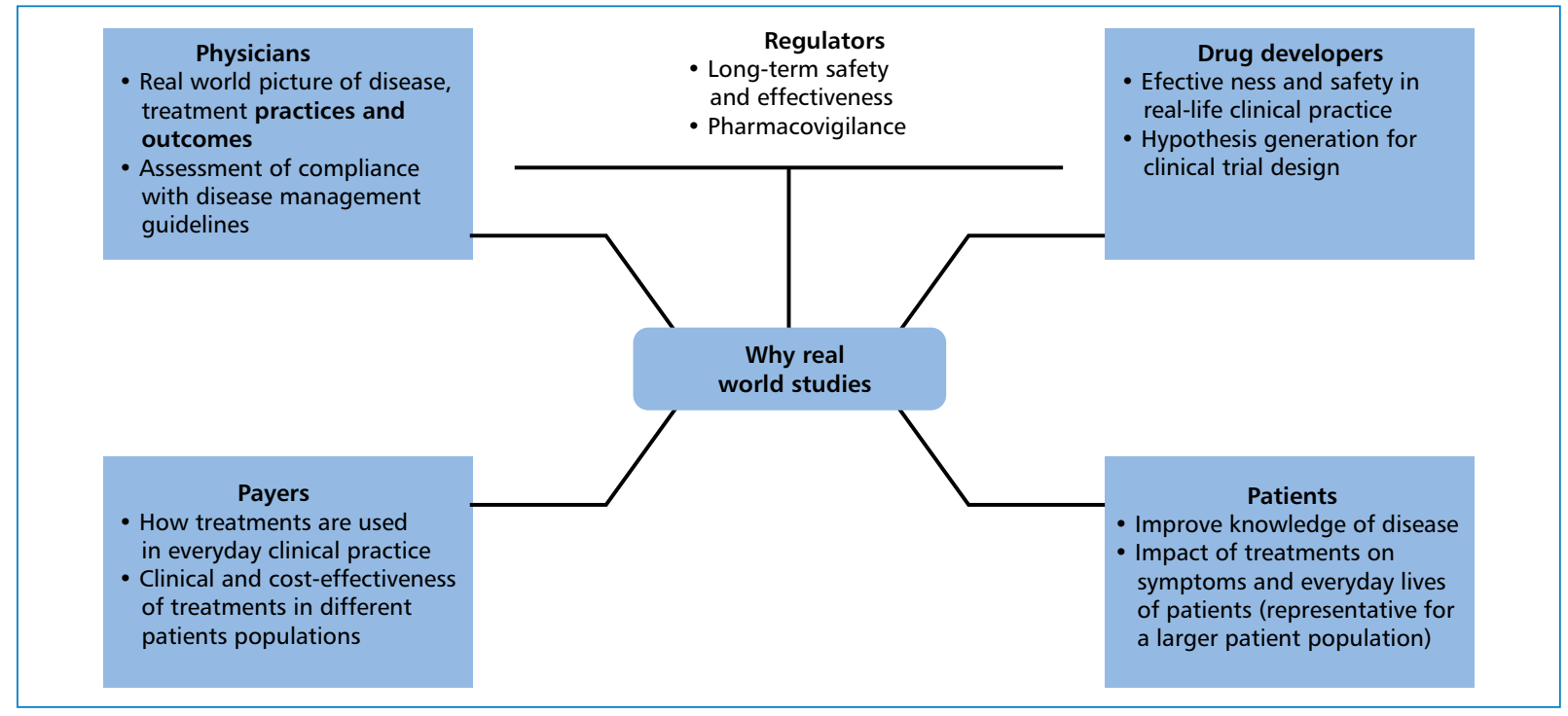

Figure 3. Value of real-word studies 
enhanced the understanding of natural history of diseases, comparative effectiveness of treatment options, and assessment and interventions for quality of life.

Countries in the developing world are also beginning to adopt RWE. Though at an incipient stage in emerging economies, use of RWE has gained momentum over the past five years growing at a rate of $11 \%$ a year [44]. China has used RWE to develop evidencebased guidelines for percutaneous coronary interventions [45]. India is adopting RWE for advancements in healthcare. However, there are several challenges including lack of patient- centricity, poor data quality, and incompleteness of data [46].

Challenges with RWE studies in India are attributable to general lack of data at majority of healthcare practices, large data sets with uncertain quality, diversity in information, lack of financial and organizational support for real-world studies, lack of trained workforce and inadequate expertise in research methodologies, and the absence of explicit policy framework [8]. There are currently no regulations for the design and conduct of real-world studies in India.

\section{Limitations of real-world evidence}

There are several limitations of RWE studies, such as [47]:

1. Limitations of access: Some datasets may require a fee for access which limits the adoption of the datasets.

2. Inconsistent coverage of healthcare settings: Majority of available healthcare databases cover outpatient settings. Inpatient settings, where most novel medications are used, remain unrepresented in EHDs $[23,48]$.

3. Validation: Validation of data elements strengthens the contribution of datasets to evidence generation. In a study of 34 EHDs in Europe, only $50 \%$ had at least one validation study published. Available datasets lack a consistent and systematic validation.

4. Uncertain quality: Accuracy and completeness of datasets is a common limitation for generation of RWE [8]. Available datasets are heterogenous and are not a dependable source for some diseasespecific variables like laboratory investigations data and biomarker data. Data on comedications, comorbidities and safety are scarce [23].

5. Confounding: Confounding factors are difficult to adjust in RWD. This may make it difficult to explain some observed effects [2].

6. Others: Integrity and interoperability are lacking in EHDs.

\section{Summary}

RWD are collected outside the controlled constraints of conventional randomised clinical trials to evaluate what is happening in normal clinical practice. RWE is structures evidence used to support regulatory decisions for approval of treatments. RWE can inform the community on optimal care, help to identify the needs and gaps in treatment, and enable an assessment of quality of care. The use of RWD may also change throughout the lifecycle of a product, particularly post authorization.

A new era of health care innovation could be unleashed by merging multiple sources of patient data to generate RWE. Evidence from "real-world" practice and utilization outside of clinical trials - is increasingly being seen as a way to tailor healthcare decisions and guide policies. Current guidelines on RWE are limited and are areas of high unmet need. Healthcare regulators should inspire the development and access of complete, consistent, accurate, secure, unfragmented, homogenous, validated, and transparent datasets to stimulate the use of data for the purpose of research, especially in developing countries.

\section{Key points}

- Source of RWE - Diverse data types such as, observational studies, current clinical practice, patient-reported experiences

- Relevance of RWE - Complement and support data from RCTs, describe outcomes representative of an everyday clinical setting

- Use of RWE - Identify disease burden and unmet medical needs, treatment reality, compare clinical effectiveness and safety

\section{Key message}

RWE is not just "Key Data" rather an integration of multiple sources of data used to tailor healthcare decision making. In the present scenario there is a distinct need to establish and develop relevant and valid datasets especially in developing countries. Active participation of healthcare regulators is pertinent to train the workforce.

\section{Acknowledgement}

All named authors meet the International Committee of Medical Journal Editors (ICMJE) criteria for authorship for this manuscript, take responsibility for the integrity of the work, and have given final approval for the version to be published.

Medical writing and editorial support in the preparation of this article was provided by Dr. Tarveen Jandoo 
and Dr. Punit Srivastava. Support for this assistance was funded by Novo Nordisk, India.

\section{Conflict of interest}

The authors declare no conflict of interest.

\section{REFERENCES:}

1. Khosla S, White R, Medina J, et al. Real world evidence (RWE) - a disruptive innovation or the quiet evolution of medical evidence generation? F1000Res. 2018; 7: 111, doi: 10.12688/ f1000research.13585.2, indexed in Pubmed: 30026923.

2. Sherman RE, Anderson SA, Dal Pan GJ, et al. Real-World Evidence - What Is It and What Can It Tell Us? N Engl J Med. 2016; 375(23): 2293-2297, doi: 10.1056/NEJMsb1609216, indexed in Pubmed: 27959688.

3. Makady A, de Boer A, Hillege H, et al. (on behalf of GetReal Work Package 1). What Is Real-World Data? A Review of Definitions Based on Literature and Stakeholder Interviews. Value Health. 2017; 20(7): 858-865, doi: 10.1016/j.jval.2017.03.008, indexed in Pubmed: 28712614.

4. USFDA. Framework for FDA's Real-World Evidence Program December 2018. https://www.fda.gov/media/120060/download. (11.10.2019)

5. Real-World Evidence. U.S. Food and Drug Administration. https:// www.fda.gov/science-research/science-and-research-specialtopics/real-world- evidence (12.10.2019).

6. Mulder $R$, Singh $A B$, Hamilton $A$, et al. The limitations of using randomised controlled trials as a basis for developing treatment guidelines. Evid Based Ment Health. 2018; 21(1): 4-6, doi: 10.1136/eb-2017-102701, indexed in Pubmed: 28710065.

7. Cartwright N, Munro E. The limitations of randomized controlled trials in predicting effectiveness. J Eval Clin Pract. 2010; 16(2): 260-266, doi: 10.1111/j.1365-2753.2010.01382.x, indexed in Pubmed: 20367845

8. Bhatt A. Conducting real-world evidence studies in India. Perspect Clin Res. 2019; 10(2): 51-56, doi: 10.4103/picr.PICR_8_19, indexed in Pubmed: 31008069.

9. Kim SY. Efficacy versus Effectiveness. Korean J Fam Med. 2013; 34(4): 227, doi: 10.4082/kjfm.2013.34.4.227, indexed in Pubmed: 23904951.

10. Klonoff DC. The New FDA Real-World Evidence Program to Support Development of Drugs and Biologics. J Diabetes Sci Technol. 2020; 14(2): 345-349, doi: 10.1177/1932296819832661, indexed in Pubmed: 30862182.

11. Dang $A$, Vallish BN. Real world evidence: An Indian perspective. Perspect Clin Res. 2016; 7(4): 156-160, doi: 10.4103/22293485.192030, indexed in Pubmed: 27843789.

12. Tibaldi JM, Hansen BB, Wolden ML, et al. Real-World Assessment of Clinical Effectiveness when Switching to Insulin Degludec from Another Basal Insulin among Type 2 Diabetes Patients in the U.S. Diabetes. 2017; 66(Supl. 1): A261.

13. Sun X, Tan J, Tang Li, et al. Real world evidence: experience and lessons from China. BMJ. 2018; 360: j5262, doi: 10.1136/bmj. j5262, indexed in Pubmed: 29437644.

14. Kalra S, Gupta Y. Planning a research project: The seven As checklist. J Pak Med Assoc. 2016; 66(12): 1515-1516, indexed in Pubmed: 27924957.

15. Harashima SI, Nishimura A, Inagaki N. Attitudes of patients and physicians to insulin therapy in Japan: an analysis of the Global Attitude of Patients and Physicians in Insulin Therapy study. Expert Opin Pharmacother. 2017; 18(1): 5-11, doi: 10.1080/14656566.2016.1260547, indexed in Pubmed: 27846737.

16. Siegmund T, Tentolouris N, Knudsen ST, et al. EU-TREAT study group. A European, multicentre, retrospective, non-interventional study (EU-TREAT) of the effectiveness of insulin degludec after switching basal insulin in a population with type 1 or type 2 diabetes. Diabetes Obes Metab. 2018; 20(3): 689-697, doi: 10.1111/ dom.13149, indexed in Pubmed: 29106039.

17. Price $H$, Blüher $M$, Prager $R$, et al. EXTRA study group. Use and effectiveness of a fixed-ratio combination of insulin degludec/ liraglutide (IDegLira) in a real-world population with type 2 diabetes: Results from a European, multicentre, retrospective chart review study. Diabetes Obes Metab. 2018; 20(4): 954-962, doi: 10.1111/dom.13182, indexed in Pubmed: 29205856.

18. Melzer Cohen C, Thorsted BL, Wolden ML, et al. Improved Glycemic Control Achieved by Switching to Insulin Degludec in Insulin-Treated Patients with Type 2 Diabetes in a Real-World Setting: a Non-interventional, Retrospective Cohort Study. Diabetes Ther. 2017; 8(5): 1047-1055, doi: 10.1007/s13300-017-0297-9, indexed in Pubmed: 28884311.

19. Fadini GP, Giordano C, Salvi L, et al. REFLECT Italian Study Group. Reduced Rates of Hypoglycemia in Type 1 or Type 2 Diabetes After Switching to Insulin Degludec: Results from the Italian Cohort of the ReFLeCT Study. Diabetes Ther. 2020; 11(12): 2909-2920, doi: 10.1007/s13300-020-00936-5, indexed in Pubmed: 33011923.

20. Gautier JF, Martinez L, Penfornis A, et al. Effectiveness and Persistence with Liraglutide Among Patients with Type 2 Diabetes in Routine Clinical Practice--EVIDENCE: A Prospective, 2-Year Follow-Up, Observational, Post-Marketing Study. Adv Ther. 2015; 32(9): 838-853, doi: 10.1007/s12325-015-0245-x, indexed in Pubmed: 26424330.

21. Sesti G, Bardtrum L, Dagdelen S, et al. A greater proportion of participants with type 2 diabetes achieve treatment targets with insulin degludec/liraglutide versus insulin glargine 100 units $/ \mathrm{mL}$ at 26 weeks: DUAL VIII, a randomized trial designed to resemble clinical practice. Diabetes Obes Metab. 2020; 22(5): 873-878, doi: 10.1111/dom.13957, indexed in Pubmed: 31903724.

22. Pacurariu A, Plueschke $K$, McGettigan $P$, et al. Electronic healthcare databases in Europe: descriptive analysis of characteristics and potential for use in medicines regulation. BMJ Open. 2018; 8(9): e023090, doi: 10.1136/bmjopen-2018-023090, indexed in Pubmed: 30185579.

23. Asche CV, Zografos P, Norlin JM, et al. Evaluation of Resource Utilization and Treatment Patterns in Patients with Actinic Keratosis in the United States. Value Health. 2016; 19(2): 239-248, doi: 10.1016/j.jval.2015.11.014, indexed in Pubmed: 27021759.

24. Khunti K, Wolden ML, Thorsted BL, et al. Clinical inertia in people with type 2 diabetes: a retrospective cohort study of more than 80,000 people. Diabetes Care. 2013; 36(11): 3411-3417, doi: 10.2337/dc13-0331, indexed in Pubmed: 23877982.

25. Gliklich RE, Dreyer NA, Leavy MB, editors. Registries for Evaluating Patient Outcomes: A User's Guide [Internet]. 3rd edition. Rockville (MD): Agency for Healthcare Research and Quality (US); 2014 Apr. Report No.: 13 (14)-EHC111.

26. Lacasse $Y$, Krishnan JA, Maltais F, et al. Patient registries for home oxygen research and evaluation. Int J Chron Obstruct Pulmon Dis. 2019; 14: 1299-1304, doi: 10.2147/COPD.S204391, indexed in Pubmed: 31417247.

27. Ziemssen T, Rothenbacher D, Kuhle J, et al. [Real-world evidence : Benefits and limitations in multiple sclerosis research]. Nervenarzt. 2017; 88(10): 1153-1158, doi: 10.1007/s00115-017-0387-y, indexed in Pubmed: 28776214

28. Ma H, Russek-Cohen E, Izem R, et al. Sources of Safety Data and Statistical Strategies for Design and Analysis: Transforming Data Into Evidence. Ther Innov Regul Sci. 2018; 52(2): 187-198, doi: 10.1177/2168479018755085, indexed in Pubmed: 29714524.

29. Fawzy AM, Yang WY, Lip GYh. Safety of direct oral anticoagulants in real-world clinical practice: translating the trials to everyday clinical management. Expert Opin Drug Saf. 2019; 18(3): 187-209, doi: 10.1080/14740338.2019.1578344, indexed in Pubmed: 30712419

30. Brod $\mathrm{M}$, Wolden $\mathrm{M}$, Christensen $\mathrm{T}$, et al. A nine country study of the burden of non-severe nocturnal hypoglycaemic events on diabetes management and daily function. Diabetes Obes 
Metab. 2013; 15(6): 546-557, doi: 10.1111/dom.12070, indexed in Pubmed: 23350726.

31. Irving $E$, van den Bor R, Welsing $P$, et al. GetReal Work Package 3. Series: Pragmatic trials and real world evidence: Paper 7. Safety, quality and monitoring. J Clin Epidemiol. 2017; 91: 6-12, doi: 10.1016/j.jclinepi.2017.05.004, indexed in Pubmed: 28502812.

32. Ford I, Norrie J. Pragmatic Trials. N Engl J Med. 2016; 375(5): 454-463, doi: 10.1056/NEJMra1510059, indexed in Pubmed: 27518663.

33. Alphs L, Benson C, Cheshire-Kinney K, et al. Real-world outcomes of paliperidone palmitate compared to daily oral antipsychotic therapy in schizophrenia: a randomized, open-label, review boardblinded 15-month study. J Clin Psychiatry. 2015; 76(5): 554-561, doi: 10.4088/JCP.14m09584, indexed in Pubmed: 25938474.

34. Lin D, Thompson-Leduc P, Ghelerter I, et al. Real-World Evidence of the Clinical and Economic Impact of Long-Acting Injectable Versus Oral Antipsychotics Among Patients with Schizophrenia in the United States: A Systematic Review and Meta-Analysis. CNS Drugs. 2021; 35(5): 469-481, doi: 10.1007/s40263-021-00815-y, indexed in Pubmed: 33909272.

35. Przepiorka D, Ko CW, Deisseroth A, et al. FDA Approval: Blinatumomab. Clin Cancer Res. 2015; 21(18): 4035-4039, doi: 10.1158/1078-0432.CCR-15-0612, indexed in Pubmed: 26374073.

36. Real-world data (RWD) and real-world evidence (RWE) are playing an increasing role in health care decisions. https://www. fda.gov/science- research/science-and-research-special-topics/ real-world-evidence.

37. European Medicines Agency. ICH E9 (R1) addendum on estimands and sensitivity 5 analysis in clinical trials to the guideline on statistical 6 principles for clinical trials. 30 August 2017. EMA CHMP/ICH/436221/2017. https://www.ema.europa.eu/en/documents/scientific-guideline/draft-ich-e9-r1-addendum-estimandssensitivity-analysis-clinical-trials-guideline-statistical_en.pdf (11.10.2019)

38. PDUFA REAUTHORIZATION PERFORMANCE GOALS AND PROCEDURES FISCAL YEARS 2018 THROUGH 2022. https://www.fda. gov/media/99140/download (23.10.2019).
39. Kim HS, Lee S, Kim JuH. Real-world Evidence versus Randomized Controlled Trial: Clinical Research Based on Electronic Medical Records. J Korean Med Sci. 2018; 33(34): e213, doi: 10.3346/ jkms.2018.33.e213, indexed in Pubmed: 30127705.

40. Jarow JP, LaVange L, Woodcock J. Multidimensional Evidence Generation and FDA Regulatory Decision Making: Defining and Using "Real-World" Data. JAMA. 2017; 318(8): 703-704, doi: 10.1001/jama.2017.9991, indexed in Pubmed: 28715550.

41. Rudrapatna VA, Butte AJ. Opportunities and challenges in using real-world data for health care. J Clin Invest. 2020; 130(2): 565574, doi: 10.1172/JCI129197, indexed in Pubmed: 32011317.

42. Gliklich \& Dreyer (eds). Registries for evaluating patient outcomes: a user's guide. 2nd ed. Rockville, MD: AHRQ, 2010. https://www. ncbi.nlm.nih.gov/books/NBK49444/.

43. Hampson G, Towse A, Dreitlein WB, et al. Real-world evidence for coverage decisions: opportunities and challenges. J Comp Eff Res. 2018; 7(12): 1133-1143, doi: 10.2217/cer-2018-0066, indexed in Pubmed: 30411972.

44. Petracci F, Ghai C, Pangilinan A, et al. Use of real-world evidence for oncology clinical decision making in emerging economies. Future Oncol. 2021 [Epub ahead of print], doi: 10.2217/fon-20210425, indexed in Pubmed: 34044583.

45. Kwong JSw, Sun X. Development of local clinical practice guidelines in the real world: an evolving scene in China. Heart Asia. 2017; 9(2): e010903, doi: 10.1136/heartasia-2017-010903, indexed in Pubmed: 29467835.

46. Dang A, Vallish BN. Real world evidence: An Indian perspective. Perspect Clin Res. 2016; 7(4): 156-160, doi: 10.4103/22293485.192030, indexed in Pubmed: 27843789.

47. Kim HS, Kim JuH. Proceed with Caution When Using Real World Data and Real World Evidence. J Korean Med Sci. 2019; 34(4): e28, doi: 10.3346/jkms.2019.34.e28, indexed in Pubmed: 30686950.

48. Neubert A, Sturkenboom MC, Murray ML, et al. TEDDY Network of Excellence. Databases for pediatric medicine research in Europe-assessment and critical appraisal. Pharmacoepidemiol Drug Saf. 2008; 17(12): 1155-1167, doi: 10.1002/pds.1661, indexed in Pubmed: 18979461. 